\title{
Hedgehog signaling regulates segment formation in the annelid Platynereis
}

\author{
Nicolas Dray ${ }^{1,5,+}$, Kristin Tessmar-Raible ${ }^{2,3,+}$, Martine Le Gouar ${ }^{1,+}$, Laura Vibert ${ }^{1}$, Foteini \\ Christodoulou $^{3}$, Katharina Schipany ${ }^{2}$, Aurélien Guillou ${ }^{4}$, Juliane Zantke ${ }^{2}$, Heidi Snyman ${ }^{3}$, \\ Julien Béhague ${ }^{1,4}$, Michel Vervoort ${ }^{1,4}$, Detlev Arendt ${ }^{3}$, and Guillaume Balavoine ${ }^{1,4,{ }^{*}}$ \\ ${ }^{1}$ Centre de Génétique Moléculaire du CNRS, FRE 3144, Avenue de la Terrasse, 91189 Gif-sur- \\ Yvette, France \\ ${ }^{2}$ Max F. Perutz Laboratories, Universität Wien, Dr. Bohr-Gasse 9, 1030 Wien, Austria \\ ${ }^{3}$ European Molecular Biology Laboratory, Developmental Biology Unit, Heidelberg 69117, \\ Germany \\ ${ }^{4}$ Institut Jacques Monod, CNRS - Université Paris Diderot, 15 rue Hélène Brion, 75205 Paris \\ cedex 13, France
}

\section{Abstract}

Annelids and arthropods share a similar segmented organization of the body whose evolutionary origin remains unclear. The Hedgehog signaling pathway, prominent in arthropod embryonic segment patterning has not been shown to have a similar function outside arthropods. We show that the ligand Hedgehog, the receptor Patched, and the transcription factor Gli are all expressed in striped patterns prior to morphological segment appearance in the annelid Platynereis dumerilii. Treatments with small molecules antagonistic to Hedgehog signaling disrupt segment formation. Platynereis Hedgehog is not necessary to establish early segment patterns but is required to maintain them. The molecular similarity of segment patterning functions of the Hedgehog pathway in an annelid and in arthropods supports a common origin of segmentation in protostomes.

In the fruitfly, the axial patterning of each individual segment is controlled and maintained by two signaling pathways operating across the segment: Wnt/ $\beta$-catenin and Hedgehog (1, 2 ). The function of the Hedgehog $(\mathrm{Hh})$ pathway in segment formation is conserved in other holometabolous insects (3) and probably also in non-insect arthropods (4, 5). By contrast, mesodermal somites in vertebrates are patterned by non-homologous segment polarity genes (6). Here, we investigated the role of the Hh pathway during segment formation in an annelid representative of the third great branch of Bilaterians, Spiralians (Fig S1). The nereid Platynereis dumerilii presents two phases of segment formation: larval metamorphosis and juvenile posterior growth (Fig S2). We cloned by degenerate PCR four genes of the Hh pathway in Platynereis coding respectively for orthologs of the ligand Hedgehog $(P d u-h h)$, its receptor Patched (Pdu-ptc), the transmembrane activator Smoothened (Pdu-smo) and the transcription factor Cubitus-interruptus/Gli (Pdu-Gli) (7, predicted primary structures in Fig S3, phylogenetic trees in Fig S4). Pdu-hh, Pdu-ptc and

\footnotetext{
to whom correspondence should be addressed, balavoine.guillaume.@ijm.univ-paris-diderot.fr.

5 Present address: Department of Molecular, Cellular and Developmental Biology, Yale University, New Haven, CT 06520-8103, USA

${ }^{+}$These authors contributed equally

Supporting Online Material www.sciencemag.org Figs. S1to S13 Supporting Text Materials and Methods Additional references
} 
$P d u-G l i$ are expressed in segment polarity-like patterns strikingly reminiscent of their arthropod counterparts, i.e. in ectodermal segmental stripes visible before segment anlagen become apparent both during larval (Fig 1A, B, D, E, G, H, Fig S5A-F) and posterior growth (Fig 1C, F, I, Fig S5G-L) (detailed descriptions in Supporting Online Text). The thin stripes of $P d u$-hh expression are located at the anterior border of each segment (Fig S5G, H), mostly in the same cells as two previously identified Platynereis segment polarity-like genes orthologous to engrailed (8) and NK4/tinman (9). The broader stripes of Pdu-ptc are centered on these $P d u-h h / e n / N K 4$ stripes spreading on each side of the segment border (Fig S5I, J) while Pdu-Gli shows a complementary pattern in the middle of each future segment (Fig S5K, L). Pdu-smo does not show distinctive patterns in whole mount in situ hybridization (WMISH) until the 3-segmented larva when it is expressed predominantly in the pharyngeal area (Fig S6). Real-time PCR measurements nevertheless show that Pdu-smo is expressed at low, presumably ubiquitous levels early on in trochophore development and that maternal mRNAs are present in fertilized eggs (Fig S6), similar to the early embryos of the fruitfly and vertebrates (10-12).

These expression patterns suggest that the Hh pathway is involved in segmental patterning in Platynereis. However, striped expressions of Hh pathway genes appears distinctly later during trochophore development (Fig S5A-F) than those of the previously identified Platynereis segment polarity-like genes Pdu-en (8), Pdu-NK4 and Pdu-Lbx (9). Thus the Hh pathway might play a late role in segment formation, in shaping the individual segments after the specification of the segmental pattern by other genes. To test this hypothesis, we used two small molecules, cyclopamine (13) and SANT-1 (14), known for antagonizing Hh signaling in vertebrates by binding to Smo proteins (7). We defined three time windows for treatments (Fig S7): an early trochophore window before any component of the Hh pathway is distinctly expressed in stripes (20 to $30 \mathrm{hpf}$ ), a mid-trochophore window when $P d u-h h$, $P d u-G l i$ and $P d u-p t c$ are all expressed in stripes (32 to $48 \mathrm{hpf}$ ) and a late trochophore window (54 hpf onward) when striped expression for Pdu-ptc and Pdu-Gli fade, but still a few hours before the larva starts to elongate and to form externally visible segments (15). Mid-trochophore treatments with increasing doses of cyclopamine or SANT-1 on mixed batches of trochophores coming from several females gave remarkably similar abnormal larvae (Fig S8.I and II). They fail to develop properly shaped segments at 3 days: They do not elongate properly, remaining ovoid in shape (Fig 2 A, B, Fig S8.II.A-C); Segmental grooves do not appear (Fig 2A, B, yellow arrows) and segmental trochae (circular ciliary belts borne by larval segments) are reduced (Fig S8.II.D-F); Appendage outgrowths and chaetae do not appear (Fig S8.II.A-C). In addition, the axonal scaffolds of the ventral nerve cord and segmental peripheral nervous system (Fig 2C, D, Fig S8.II.D-F) both appear underdeveloped and disorganized. In place of the metameric peripheral nerves (red asteriks), an asymmetric proliferation of axons projecting haphazardly to the periphery forms, indicating that the segmental pattern of the nervous system is lost. The effects of two unrelated drugs and of different treatment time windows on embryos suggest that the phenotypes obtained are caused by blocking Hh signaling (Fig S8, Supporting Online Text). In particular, the phenotypes cannot be due to a general toxicity of the drugs since late trochophore treatments at a time of massive proliferation and morphogenesis cause few defects (Fig S8.II.G-K). We also tested the role of Pdu-smo on posterior segment addition by treating individuals with cyclopamine after caudal regeneration. One half (12/24) of treated individuals show regenerated posterior parts with fewer, abnormally shaped segment anlagen and reduced segmental grooves (Fig S12).

To analyze further the effects of cyclopamine on the segmental pattern, we looked at the expression patterns of the previously known segment polarity-like genes in cyclopamine treated larvae (7). To take into account a strong maternal effect polymorphism in the response to cyclopamine (Supporting Online Text and Fig S11A), we carefully selected only 
those batches of embryos that presented medium and strong morphological phenotypes (scored in 3-day larvae) for the gene expression study. Expression patterns for medium strength morphological phenotypes are presented in Fig 2E-L and Fig S10. Complete results including 1 or 2 repeat experiments for each gene indicate that the effects of cyclopamine on gene expression correlate well with the strength of the phenotype in each same-female batch studied (Fig S11B). Early-stage cyclopamine treatment has no effect on the striped expressions of Pdu-en, Pdu-NK4 and Pdu-Lbx (Fig S10 and Fig S11B) seen in $30 \mathrm{hpf}$ larvae, showing that $P d u-S m o$ does not act on the segmental pattern independently of the morphogen $P d u-H h$ and that the Hh pathway does not intervene in the initial laying of the segmental pattern. By contrast, mid-trochophore cyclopamine treatment at high dose leads to a complete or almost complete loss of striped expression for Pdu-hh, Pdu-en, Pdu-Wnt1 (Fig 2E, F, G, respectively), showing that the segmental pattern is largely affected well before metamorphosis. Pdu-ptc is also downregulated (Fig S9) as it is observed in $h h$-mutant flies (16) and vertebrates lacking shh expression (17). Pdu-Gli (Fig 2I) stripes are mostly persistent even at high doses of cyclopamine. The specificity of the effects of midtrochophore cyclopamine treatment on the patterning of the segmental ectoderm is demonstrated by the persistence of gene expression in tissues other than segmental ectoderm. Hence the expression of $P d u-e n$ and $P d u-L b x$ in segmental mesoderm (Fig $2 \mathrm{~F}$ and $2 \mathrm{~K}$, red arrows), the stomodeal expression of $P d u-h h$ and $P d u-W n t 11$ (Fig $2 \mathrm{E}$ and $2 \mathrm{H}$, blue asterisks) and the pygidial expression of $P d u-W n t 11$ and $P d u-C d x$ (Fig $2 \mathrm{H}$ and $2 \mathrm{~L}$, green asterisks) are maintained whereas the proctodeal expression of $P d u$-Wnt 1 is slightly enlarged (Fig 2G, green asterisks).

The effect of cyclopamine on $P d u-h h$ stripes must be indirect if we suppose that the direct targets of Hh signaling are only those cells expressing Pdu-Gli, away from segment borders. In Drosophila (Fig 3), wingless ( $\mathrm{wg}$ ) expression is maintained by Hh signaling in the anterior compartment just anterior to the en stripe whereas $\mathrm{wg}$ signaling is necessary to maintain en and $h h$ striped expression in the posterior compartment (18). In Platynereis, $P d u$-Wnt1, the orthologue of Drosophila wingless (8), is expressed just anterior to Pdu-enl $P d u-h h$ stripes on the other side of segmental grooves. Its strong downregulation by cyclopamine (Fig 2G) is consistent with a loop of regulation similar to the one known in Drosophila. Ladybird, the fly gene orthologous to $P d u-L b x$ is expressed in epidermal stripes overlapping wingless stripes in the anterior compartment of the epidermis and is positively regulated by wingless (19). By contrast, cyclopamine treatment in the annelid does not abolish completely $P d u-L b x$ stripes in trochophores (Fig $2 \mathrm{~K}$ ), suggesting that other factors are involved in its maintenance. Another difference between the annelid and the fly is that $P d u-W n t 1$ is not expressed in complete circular stripes in the trochophore (it does during posterior growth) but only in the lateral parapodial field (Fig 2G). It was thus interesting to look at $P d u-S m o$ regulation of other $W n t$ genes potentially involved in segment formation. $P d u-W n t 11$ is expressed in thick stripes in the posterior halves of segments and is strongly downregulated by cyclopamine (Fig $2 \mathrm{H}) . P d u-W n t 5$ is expressed in the anterior halves of segments and is by contrast much more resistant to cyclopamine (Fig 2J).

Our work demonstrates the involvement of the Hh pathway in segment formation outside of arthropods. It comes in contrast with earlier studies of $h h$ orthologues in annelid species (20, 21). The comparison of the effect of Hh signaling inhibition in Platynereis and in insects, i.e. the fruitfly and the coleopteran Tribolium (3), reveals extensive similarities. As in insects, Platynereis $\mathrm{Hh}$ presumably diffuses anteriorly to maintain $W n t$ signaling anterior to the $h h$ expressing cells (Fig 3), which may in turn be crucial to maintain segment boundary gene expressions, including Pdu-hh. As in insects, Hh is not necessary in the initial setting of the segmental pattern but is required to maintain this pattern before the morphological appearance of segments. The comparison of segment polarity gene patterns between Platynereis and arthropods (Fig 3) reveals four independent players with remarkably similar 
expressions: engrailed, ladybird/Lbx, the Hh pathway and Wnt1/wingless signaling. The most likely explanation of these similarities is that these genes were already playing similar roles in a metameric protostome ancestor. The alternative explanation would be a parallel recruitment of these genes for similar functions in annelids and arthropods. Since they are not known to be part of a conserved core regulatory network or "kernel" (22) that might have been co-opted en bloc, each gene would have been recruited independently, which seems unlikely. Altogether, these four players are expressed in the same spatial relationship across the annelid segment boundary as they are across the parasegmental boundary in arthropods (Fig 3). This suggests that these two boundaries are homologous as was proposed earlier (8). Therefore, the segmented exoskeleton of the arthropods must have evolved out of frame with the ancestral protostome segmentation (Fig S13). This ancestral protostome segmentation is nowadays "recapitulated" as parasegmental patterning in the embryos of extant arthropods.

\section{Supplementary Material}

Refer to Web version on PubMed Central for supplementary material.

\section{Acknowledgments}

We acknowledge the following agencies for funding: the CNRS, University Paris-Diderot and the ANR (grant BLAN-0294), the MFPL funds and the FWF START award (\#AY0041321), the French Research Ministry and the FRM for fellowships (ND), the Boehringer Ingelheim Foundation and the Marie Curie RTN ZOONET (MRTNCT-2004-005624) for fellowships (KTR). Genbank accession numbers for the gene sequences newly identified in this study are HM179271 to HM 179276.

\section{References}

1. Hatini V, DiNardo S. Trends Genet. 2001; 17:574. [PubMed: 11585663]

2. Sanson B. EMBO Rep. 2001; 2:1083. [PubMed: 11743020]

3. Farzana L, Brown SJ. Dev Genes Evol. 2008; 218:181. [PubMed: 18392879]

4. Pechmann M, McGregor AP, Schwager EE, Feitosa NM, Damen WG. Proc Natl Acad Sci U S A. 2009; 106:1468. [PubMed: 19147844]

5. Simonnet F, Deutsch J, Queinnec E. Dev Genes Evol. 2004; 214:537. [PubMed: 15365834]

6. Holley SA. Dev Dyn. 2007; 236:1422. [PubMed: 17486630]

7. Materials and methods are available as supporting material on Science Online.

8. Prud'homme B, et al. Curr Biol. 2003; 13:1876. [PubMed: 14588243]

9. Saudemont A, et al. Dev Biol. 2008; 317:430. [PubMed: 18343360]

10. van den Heuvel M, Ingham PW. Nature. 1996; 382:547. [PubMed: 8700230]

11. Alcedo J, Ayzenzon M, Von Ohlen T, Noll M, Hooper JE. Cell. 1996; 86:221. [PubMed: 8706127]

12. Chen W, Burgess S, Hopkins N. Development. 2001; 128:2385. [PubMed: 11493557]

13. Chen JK, Taipale J, Cooper MK, Beachy PA. Genes Dev. 2002; 16:2743. [PubMed: 12414725]

14. Chen JK, Taipale J, Young KE, Maiti T, Beachy PA. Proc Natl Acad Sci U S A. 2002; 99:14071. [PubMed: 12391318]

15. Steinmetz PR, Zelada-Gonzales F, Burgtorf C, Wittbrodt J, Arendt D. Proc Natl Acad Sci U S A. 2007; 104:2727. [PubMed: 17301244]

16. Hidalgo A, Ingham P. Development. 1990; 110:291. [PubMed: 2081466]

17. Concordet JP, et al. Development. 1996; 122:2835. [PubMed: 8787757]

18. DiNardo S, Heemskerk J, Dougan S, O’Farrell PH. Curr Opin Genet Dev. 1994; 4:529. [PubMed: 7950320]

19. Jagla K, et al. Development. 1997; 124:91. [PubMed: 9006070]

20. Kang D, et al. Development. 2003; 130:1645. [PubMed: 12620988]

21. Seaver EC, Kaneshige LM. Dev Biol. 2006; 289:179. [PubMed: 16330020] 
22. Davidson EH, Erwin DH. Science. 2006; 311:796. [PubMed: 16469913] 


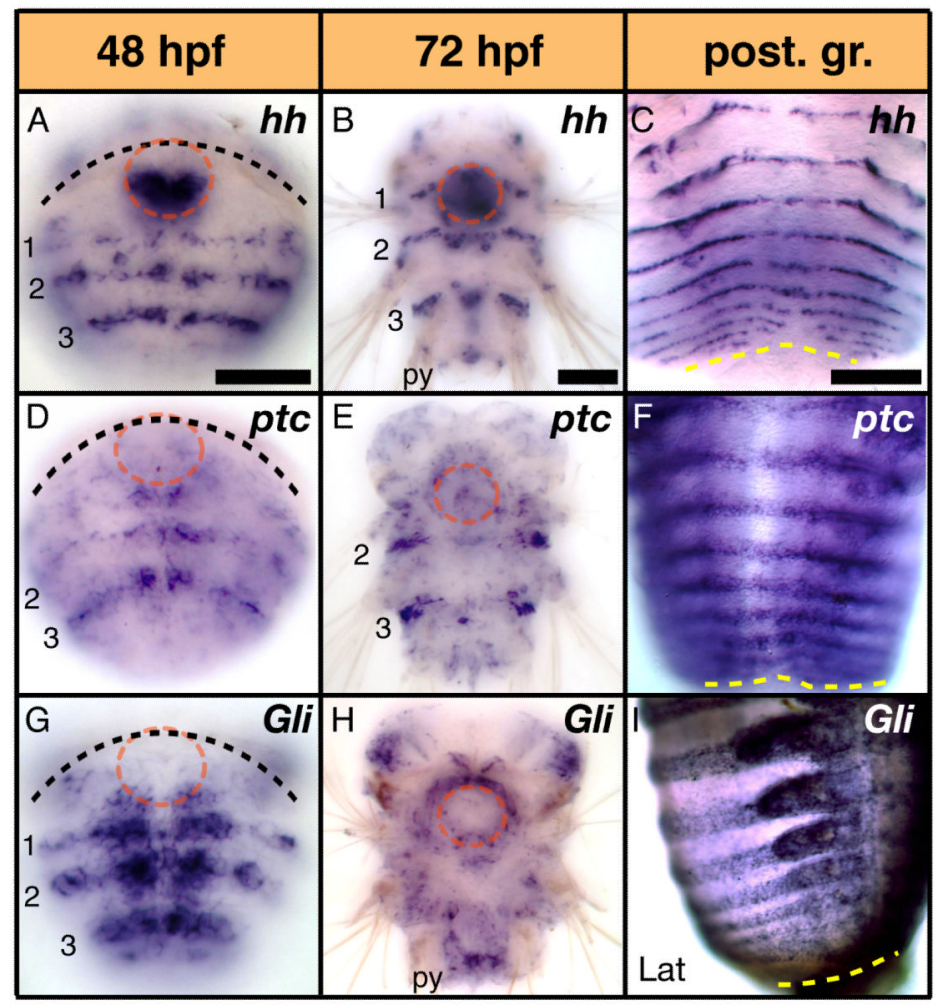

Figure 1.

Expression patterns of $h h(\mathrm{~A}-\mathrm{C})$, ptc (D-F) and Gli (G-I) orthologues in Platynereis late trochophores (48 hpf), early three-segmented larvae (72 hpf) and during posterior growth. All views are ventral except $\mathrm{I}$, lateral right (Lat). Anterior or apical is up. Black dashed line: prototroch of larvae. The stomodeum is delimitated by a red dashed circle. Larval segment anlagen are numbered 1-3. Posterior growth panels (post. gr., C, F, I) show patterns in posterior regenerates one week after caudal amputation, in front of the thin segment addition zone (yellow dashed line). Scale bars represent $50 \mu \mathrm{m}$. py: pygidium. 


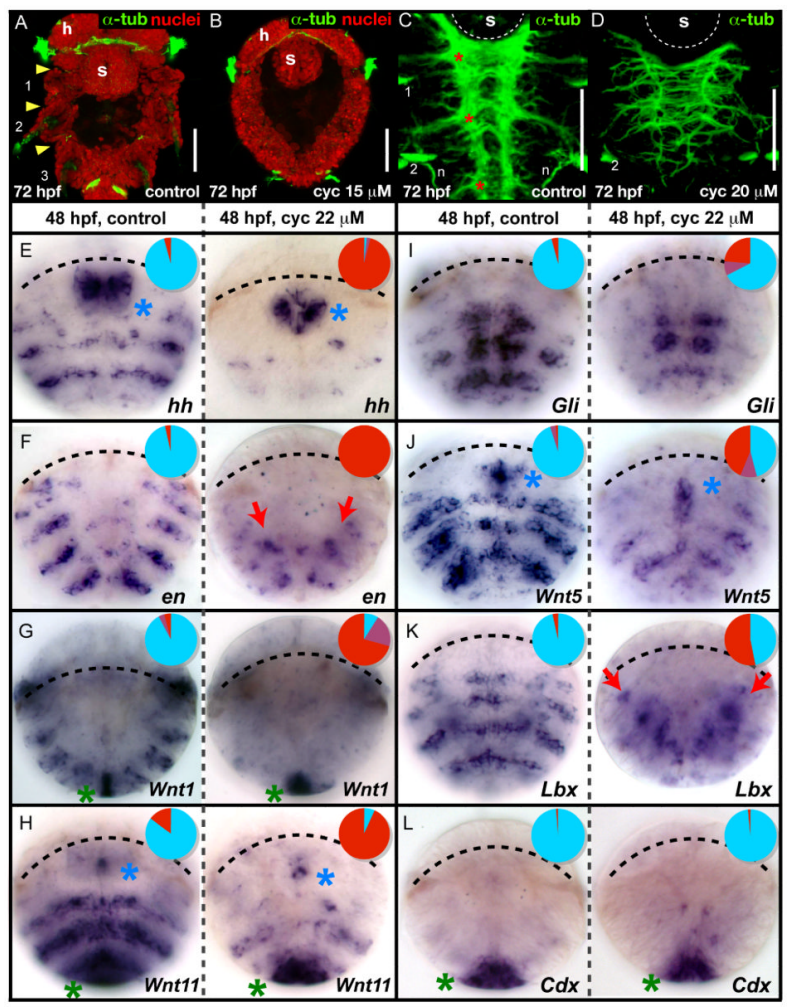

Figure 2.

Effects of mid-trochophore stage cyclopamine treatments on the development of Platynereis larvae. (A-D) 3-day larvae stained with an anti-acetylated tubulin antibody revealing the ciliary belts (trochae) (A, B) and the axonal scaffold of the nervous system (C, D). (A, B) are mid-body frontal confocal sections of Hoechst 33342 stained larvae whereas $(C, D)$ are ventral frontal confocal sections. (E-L) Effects of cyclopamine treatment on the expressions of segment polarity-like genes and $P d u-C d x$, a pygidial ectoderm marker. The first and second columns show expression in untreated larvae and in the majority of treated larvae, respectively. Pie graphs show the frequencies of abnormal expressions with normal in blue, abnormal in red and the maternal batch variability in violet where available. Small fractions of abnormal expressions in the untreated control reflect both a small proportion of spontaneous abnormal development and the probe-dependent imperfect sensitiveness of the WMISH protocol. Prototrochs: dashed black line. Stomodeum: white dashed circle and "s". p: prototroch. 1, 2, 3: larval segments in A, B, segmental trochae in C, D. Yellow arrows: segmental grooves. Red asteriks: main lateral nerve roots. n: protonephridium. h: head lobes. Red arrows, blue asterisks and green asterisks indicate persistent mesodermal, stomodeal or pygidial/proctodeal expressions, respectively. 


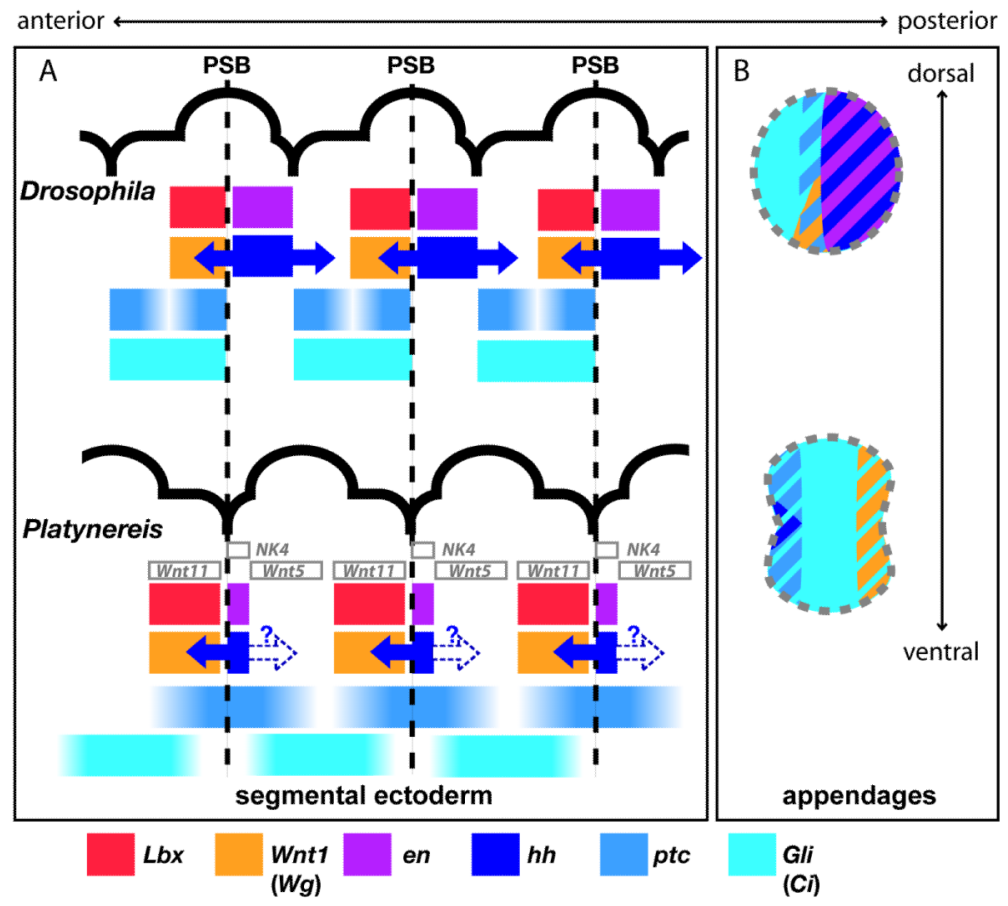

Figure 3.

(A) Comparison of the antero-posterior extension of striped expression patterns of proven and presumed segment polarity orthologous genes in Platynereis and Drosophila. Segment boundaries and limb anlagen positions are represented with a schematic body wall section in black. Orthologous genes are color-coded whereas segment polarity-like genes specific to Platynereis are represented by gray rectangles. The morphogenetic actions of the diffusible Hh proteins are represented with blue arrows. A white arrow with question mark indicates that $\mathrm{Hh}$ action on the anterior parts of annelid segments is not proven in this study. Unlike in fly, Pdu-ptc is expressed in en/hh positive cells and Pdu-Gli expression does not abut en/hh positive cells in Platynereis. (B) Comparison of expression patterns of some of these segment polarity-like genes in the early stages of appendage formation. The homology of annelid segments with arthropod parasegments implies that annelid parapodia do not correspond in embryonic position with the arthropod appendages, suggesting that these appendages do not share a common origin in the protostome ancestor. PSB: parasegmental boundary. 